\title{
Stimulated Emission Depletion Microscopy Resolves Individual Nitrogen Vacancy Centers in Diamond Nanocrystals
}

\author{
Silvia Arroyo-Camejo, ${ }^{\dagger, \perp, *}$ Marie-Pierre Adam, ${ }^{\neq, \perp}$ Mondher Besbes, ${ }^{\S}$ Jean-Paul Hugonin, ${ }^{\S}$ Vincent Jacques, \\ Jean-Jacques Greffet, ${ }^{\S}$ Jean-François Roch, ${ }^{\ddagger}$ Stefan W. Hell, ${ }^{+, *}$ and François Treussart ${ }^{\ddagger}, *$ \\ ${ }^{\dagger}$ Department of NanoBiophotonics, Max Planck Institute for Biophysical Chemistry, 37077 Goettingen, Germany, ${ }^{\ddagger}$ Laboratoire Aimé Cotton, CNRS, Université Paris \\ Sud and ENS Cachan, bât. 505, campus universitaire d'Orsay, F91405 Orsay cedex, France, and 'Laboratoire Charles Fabry, CNRS, Institut d'Optique and Université \\ Paris-Sud, 2 av A. Fresnel, F91127 Palaiseau, France. ${ }^{\perp}$ These authors have equally contributed to this work.
}

\begin{abstract}
Nitrogen-vacancy (NV) color centers in nanodiamonds are highly promising for bioimaging and sensing. However, resolving individual NV centers within nanodiamond particles and the controlled addressing and readout of their spin state has remained a major challenge. Spatially stochastic superresolution techniques cannot provide this capability in principle, whereas coordinate-controlled super-resolution imaging methods, like stimulated emission depletion (STED) microscopy, have been predicted to fail in nanodiamonds. Here we show that, contrary to these predictions, STED can resolve single NV
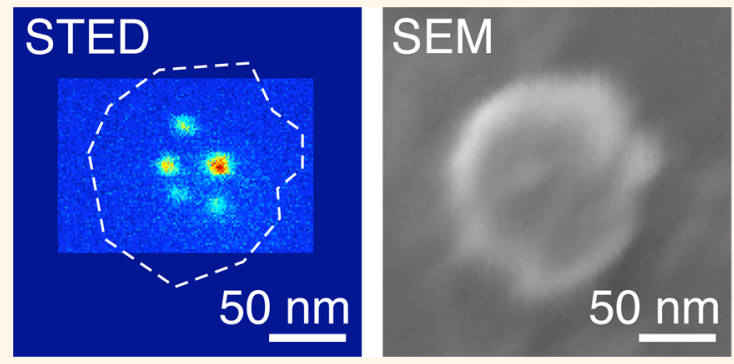
centers in $40-250 \mathrm{~nm}$ sized nanodiamonds with a resolution of $\approx 10 \mathrm{~nm}$. Even multiple adjacent NVs located in single nanodiamonds can be imaged individually down to relative distances of $\approx 15 \mathrm{~nm}$. Far-field optical super-resolution of NVs inside nanodiamonds is highly relevant for bioimaging applications of these fluorescent nanolabels. The targeted addressing and readout of individual NV ${ }^{-}$spins inside nanodiamonds by STED should also be of high significance for quantum sensing and information applications.
\end{abstract}

KEYWORDS: subdiffraction resolution microscopy · STED · nanoscopy $\cdot$ nitrogen-vacancy color center · diamond nanocrystals · finite element method

$\mathrm{N}$ itrogen-vacancy (NV) color centers in diamond nanocrystals (nanodiamond, ND) have emerged as promising candidates for various bioimaging applications due to their remarkably stable photoluminescence and owing to the distinct feature of the negatively charged $\mathrm{NV}^{-}$center to enable optically detected magnetic resonance (ODMR) measurements of the $\mathrm{NV}^{-}$electron spin. Its sensitivity to magnetic ${ }^{1,2}$ and electric fields, ${ }^{3}$ as well as sensitivity to temperature, ${ }^{4}$ makes the $\mathrm{NV}^{-}$a highly versatile sensor for bioapplications. Combining this atom-sized solid-state system with subdiffraction resolution optical microscopy should allow sensing the interior of cells at the nanoscale in a unique, multifunctional way. Given the potential of these particles and their application range, mapping of NV centers within individual ND has become important. Apart from super-resolution imaging, the ability to optically address and read out the spin information of individual NVs is a most desired capability.

Among the various far-field optical techniques providing resolution that is not fundamentally limited by diffraction, stimulated emission depletion (STED) microscopy has turned out to be highly suitable for imaging NV centers. In bulk diamond, record resolutions down to $6 \mathrm{~nm}^{5}$ were achieved, and in conjunction with solid immersion, a resolving power of $2.4 \mathrm{~nm}^{6}$ was demonstrated, corresponding to $1 / 323$ of the applied wavelength. However, the task to individually resolve NV centers located inside diamond crystals of subwavelength dimensions (i.e., nanodiamonds) by means of STED microscopy has not yet been demonstrated, ${ }^{7,8}$ nourishing speculations about whether it is possible at all. In fact,
* Address correspondence to sarroyo@gwdg.de shell@gwdg.de, francois.treussart@ens-cachan.fr.

Received for review August 23, 2013 and accepted November 18, 2013.

Published online November 18, 2013 $10.1021 / \mathrm{nn} 404421 \mathrm{~b}$

C) 2013 American Chemical Society 
recent simulations considering Mie scattering of a focused doughnut beam for STED on spherical NDs of various sizes had indicated that with STED microscopy or related techniques relying on a light beam featuring an intensity zero, the achievable resolution should be limited to the diameter of the ND. ${ }^{9}$ It was suggested that the intensity around the doughnut null would be homogenized inside a spherical diamond nanoparticle if only the dipolar mode is excited, thus precluding the preparation of NV centers having two different states within a single nanodiamond.

Therefore, a stochastic super-resolution scheme has recently been applied, ${ }^{10}$ whereby the ground state of the NV center has been depleted by widefield illumination such that the NV centers were transiently shelved in a metastable dark state and the remaining fluorescent centers localized by calculating the centroid of their diffraction pattern in the image. ${ }^{10}$ Called GSDIM, ${ }^{11}$ this method requires all but a single NV center within about a $250 \mathrm{~nm}$ range to be dark, in turn requiring tight control of dark state occupation, i.e., of the 'blinking'. In reality, it is difficult to control blinking such to ensure that at most a single NV center is signaling within a $250 \mathrm{~nm}$ spatial range. Since the blinking of the NV centers was uncontrolled in that experiment, ${ }^{10}$ the fulfillment of this requirement was not strictly ascertained, meaning that the NV centers found in the ND may not have been properly located or resolved. A stochastic imaging method based on time-deterministic spin-dependent fluorescence switching mediated by microwave pulses ${ }^{12}$ addresses this problem, but even in the latter, the finite ODMR line width and the stochastic nature of the ODMR line distributions leads to significant mislocalization as the density of $\mathrm{NV}^{-}$ centers per diffraction limited area increases. Furthermore, in ref 10 only $40 \%$ of the fluorescent NDs (most of which contained more than one NV) exhibited blinking, implying that the majority of NV centers could not be resolved by this technique. For these reasons, a coordinate-targeted super-resolution method such as STED that switches the fluorescence of the NV centers truly and deterministically, both temporally and spatially is more accurate and powerful. STED on NV centers provides a fluorescence switching contrast of virtually $100 \%$ compared to the only $30 \%$ contrast of the microwave assisted localization method. ${ }^{12}$ Most importantly, STED is a more universal super-resolution method, as it can super-resolve both NV charge states $\mathrm{NV}^{-}$and $\mathrm{NV}^{0}$ in bulk diamond as well as in nanodiamonds, and even at high NV densities, while the applicability of other methods is principally limited to the imaging of $\mathrm{NV}^{-}$in samples with low marker densities. ${ }^{10,12}$ Ultimately, STED has demonstrated the highest resolution of all NV imaging methods both in bulk diamond and in diamond nanocrystals. Reaching beyond the scope of pure imaging, STED enables the targeted optical addressing and readout of individual
$\mathrm{NV}^{-}$spins inside bulk diamond and nanodiamonds, which is of high significance for application in quantum sensing and information processing and cannot be performed by super-resolution techniques based on (semi)stochastic single emitter switching.

In this work we apply STED microscopy to NV centers in nanodiamonds of various shapes and size ranging from 40 to $250 \mathrm{~nm}$ and containing 1 to $\sim 20$ NV centers. We show that under these conditions, STED microscopy is capable of resolving single NV centers in nanodiamonds with a superior resolution of approximately $10 \mathrm{~nm}$ and discerning individual NV centers within nanodiamonds down to distances of approximately $15 \mathrm{~nm}$. The study is complemented by numerical simulations supporting the experimentally observed validity of the STED principle inside NDs of subwavelength diameter, in contrast to earlier predictions. $^{9}$

\section{RESULTS AND DISCUSSION}

STED Microscopy of NV Centers in Diamond. STED fluorescence microscopy provides nanoscale resolution by forcing neighboring emitters to assume the fluorescent state sequentially in time. To this end, this technique typically utilizes a doughnut-shaped STED beam that confines NV emitters to the ground state, thus transiently turning off their signaling capability. Only in a subdiffraction-sized area around the doughnut null, where the STED beam intensity is lower than the threshold of excited state depletion by stimulated emission, the NV centers are not turned off and hence still allowed to emit. This area has a diameter of $\Delta d=$ $D /\left[1+\left(I / I_{\mathrm{s}}\right)\right]^{1 / 2} \ll D$, where $D=\lambda /(2 \mathrm{NA})$ denotes the diffraction limit, $\lambda$ is the wavelength, NA is the numerical aperture of the objective lens, $l$ is the intensity of the doughnut crest, and $I_{\mathrm{s}}$ is the characteristic intensity that reduces the population probability of the fluorescent state of the NV center by half. Scanning overlapped excitation and doughnut beams across the sample forces NV centers that are just barely further apart than $\Delta d$ to emit sequentially. This sequential occupation of their signaling state allows separate detection of highly packed neighboring centers.

To explore the maximally achievable resolution of NV centers in subwavelength sized nanodiamonds, we employed a customized STED microscope optimized for imaging NV centers in diamond (see Figure 1c). Figure 1a shows the level structure of the negatively charged $\mathrm{NV}^{-}$center formed by a substitutional nitrogen atom (N) in the diamond lattice, an adjacent lattice vacancy $(\mathrm{V})$ and an additional electron charge (see cartoon image of the NV in Figure 1b). The Hamiltonian of the $\mathrm{NV}^{-}$ground state triplet in the presence of a magnetic field $\mathbf{B}$ is given by

$$
H=D\left(S_{z}^{2}-\frac{1}{3}(S(S+1))\right)+E\left(S_{x}^{2}-S_{y}^{2}\right)+g \mu_{\mathrm{B}} \mathbf{B} \cdot \mathbf{S}
$$



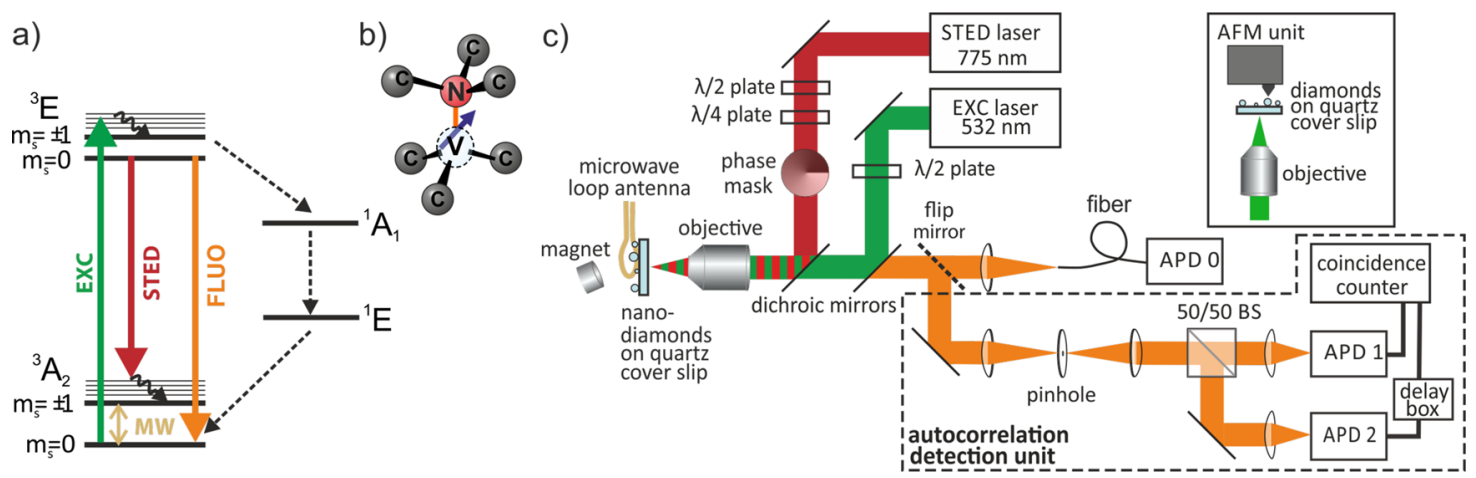

Figure 1. (a) Energy levels of the triplet (left) and singlet states (right) of the NV center and the applied excitation (EXC), deexcitation (STED) and microwave (mw) fields and the detected fluorescence (FLUO). (b) Sketch of NV center in the diamond matrix. (c) STED microscope for investigations of the resolution limit of single NV centers in diamond nanocrystals of subwavelength size (APD: avalanche photodiode in single photon counting mode), including the microwave excitation antenna next to the sample, and a Hanbury Brown and Twiss time intensity correlation measurement setup on the detection path, after the pinhole, consisting in a 50/50 beamsplitter (BS), two APD, a delay box and a coincidence counting module. The top right inset shows the combined confocal and AFM colocalization setup used for precharacterization (Supporting Information).

where $S$ is the NV electron spin, $D$ is the zero field splitting, $E$ is the strain field, $g$ is the $g$-factor of the electron, and $\mu_{\mathrm{B}}$ is the Bohr magneton. In the diamond lattice, an NV center can occupy one out of four possible orientations. ${ }^{13}$ Hence, for an NV ensemble in a diamond monocrystal, a homogeneous external magnetic field will lead to the emergence of at most four different ESR line pairs, as all NVs of the same orientation have mutually degenerate Zeeman levels. In NDs, however, the degeneracy of ESR lines of NVs with identical orientation is lifted due to the strongly varying local strain fields $E$ (cf. eq 1 ), giving almost every $\mathrm{NV}^{-}$a distinct signature in the ESR spectrum. Therefore, ESR spectra provide a valuable complementary method for the determination of the approximate number of $\mathrm{NV}^{-}$centers present in an ND.

Super-resolving Single NVs in Single NDs. Figure 2a displays both a confocal and a STED image of a nanodiamond containing a single NV center, as confirmed by the antibunching dip in the respective $g^{(2)}(\tau)$ autocorrelation function measurement (see Figure $2 c$ ). Upon application of STED microscopy, the spatial intensity profile of the NV image is reduced in full width at halfmaximum (FWHM) from $(325.8 \pm 3.5)$ to $(9.5 \pm 1.2) \mathrm{nm}$. This image inherently represents the effective point spread function (PSF) of the STED imaging system. The reported sharpening of the PSF FWHM to less than $3 \%$ of its confocal counterpart signifies a more than 34-fold resolution increase. The lateral dimensions of the nanodiamonds were readily gained by scanning electron microscopy (SEM; see Supporting Information). The SEM image of the corresponding NV host particle is displayed in Figure $2 \mathrm{~b}$ as an inset. We determined the lateral ND dimension to be approximately 45 and $100 \mathrm{~nm}$. Additionally, the vertical dimension of the ND was measured in a precharacterization step using an Atomic Force Microscope combined to a confocal microscope for simultaneous fluorescence and topography recording (Supporting Information).

Figure $2 \mathrm{~d}$ shows the scaling of the FWHM (extracted from Gaussian or Lorentzian fits to the STED images) as a function of the STED beam power. The data was taken from six NDs of various mean lateral diameters, with each of the ND containing a single NV center (SEM images of the six NDs are to be found in the Supporting Information). In fact, neither the scaling of the STED resolution with the STED beam power nor the maximum resolution obtained with an NV center shows any dependence on the ND shape or size. Moreover, at the maximum STED power applied, we routinely recorded subdiffraction resolution profiles and images of single NV centers with FWHM of 10-20 nm. Again, as can be concluded from the data shown in Figure $2 d$, these FWHM showed no dependence on the ND size.

Super-resolving Multiple NVs in Single NDs. Even when a nanosized diamond crystal contained several NV centers, we were able to resolve them with $\sim 10 \mathrm{~nm}$ resolution (FWHM). Figure 3 shows a typical set of measurements performed on a particle with a mean dimension of around $100 \mathrm{~nm}$ containing five NV centers. The STED image in Figure 3a displays 5 fluorescence spots with a FWHM of around $10 \mathrm{~nm}$ (see one-dimensional projection in Figure $3 \mathrm{~b}$ ). Figure $3 c$ is an SEM image of the same ND, showing that it is a single crystal and not an aggregate.

To illustrate the size relations, Figure $3 \mathrm{~d}$ displays an overlay of the STED image (from Figure $3 a$ ) and the SEM image (from Figure $3 c$ ) of the same ND. The colocalization uncertainty of the STED with respect to the SEM images is on the order of the nanodiamond size. In Figure 3, the absolute colocalization uncertainty is $<40 \mathrm{~nm}$. Figure 3e provides an optically detected ESR spectrum of the same ND (detected in confocal imaging mode) featuring five line pairs. In general, we observed a strong correlation between the number of 

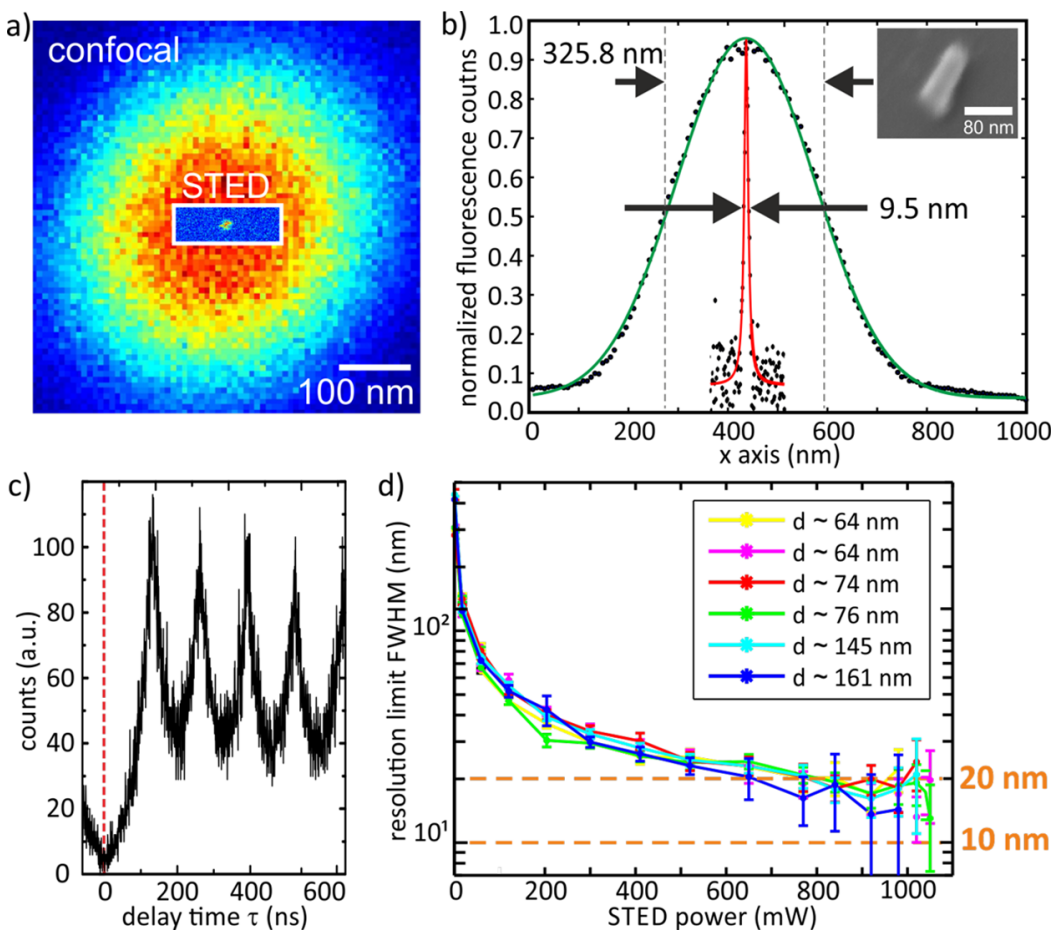

Figure 2. (a) Comparison of a confocally detected image versus a STED image in the centered inset and (b) their respective side projection of a single NV in an ND (green curve, Gaussian fit; red curve, Lorentzian fit; the inset shows an SEM image of the ND). (c) Measurement of the $g^{(2)}(\tau)$ correlation function of the same ND proving that it incorporates a single NV center. (d) Scaling of the minimal full width at half-maximum (FWHM) of single NVs in NDs of various mean dimensions $d$ in dependence on the applied STED power.
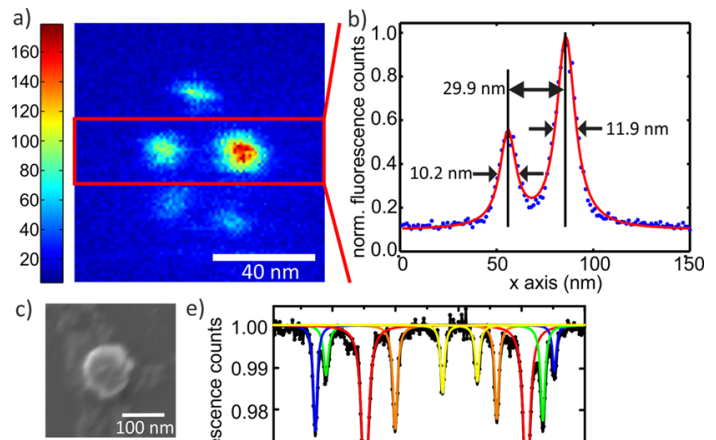

d)

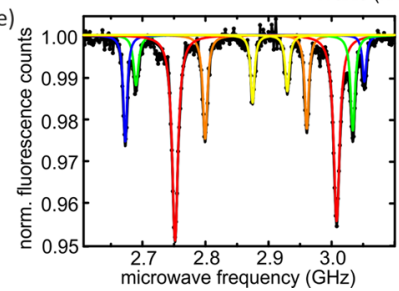

Figure 3. (a) Subdiffraction resolution STED image and (b) corresponding vertically binned STED image profile of a diamond particle with $\sim 100 \mathrm{~nm}$ diameter showing five isolated $\mathrm{NV}^{-}$centers (red curve: Lorentzian fit). (c) SEM image of the same nanodiamond and (d) overlay of the STED image and the SEM image illustrating the relative dimensions. (e) Confocally recorded ESR spectrum of the same nanodiamond showing five distinct ESR line pairs corresponding to the five $\mathrm{NV}^{-}$centers (each line pair fitted with a double Lorentzian in a separate color).

fluorescence spots in the STED images and the number of line pairs in the corresponding ESR spectra (see Supporting Information). Hence, we concluded that the isolated spots identifiable in the STED images originate from individual NV centers in the NDs.

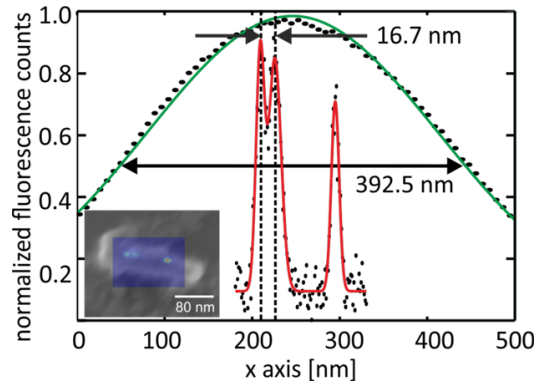

Figure 4. Confocal (green curve, Gaussian fit) and STED (red curve, triple Lorentzian fit) fluorescence profile of an exemplary nanodiamond with a mean dimension of around $210 \mathrm{~nm}$ showing three isolated $\mathrm{NV}^{-}$centers, two of which are $(16.7 \pm 1.6) \mathrm{nm}$ close (the inset displays an overlay of the STED image and the SEM image illustrating the relative dimensions).

On a different and irregularly shaped ND (lateral side lengths: 100 and $200 \mathrm{~nm}$ ) containing three NV centers, we demonstrated that we could resolve two individual centers located $(16.7 \pm 1.6) \mathrm{nm}$ apart from each other (see Figure 4). In good agreement with our demonstrated resolution of $10-20 \mathrm{~nm}$ and considering a statistical distribution of the NVs within a twodimensional projection of the ND volume (of typically 100 to $300 \mathrm{~nm}^{2}$ ), STED imaging enabled us to identify up to eight isolated NV centers in single ND with a mean dimension $<250 \mathrm{~nm}$.

Numerical Simulations. To confirm theoretically that STED microscopy is able to image NV centers in ND and 

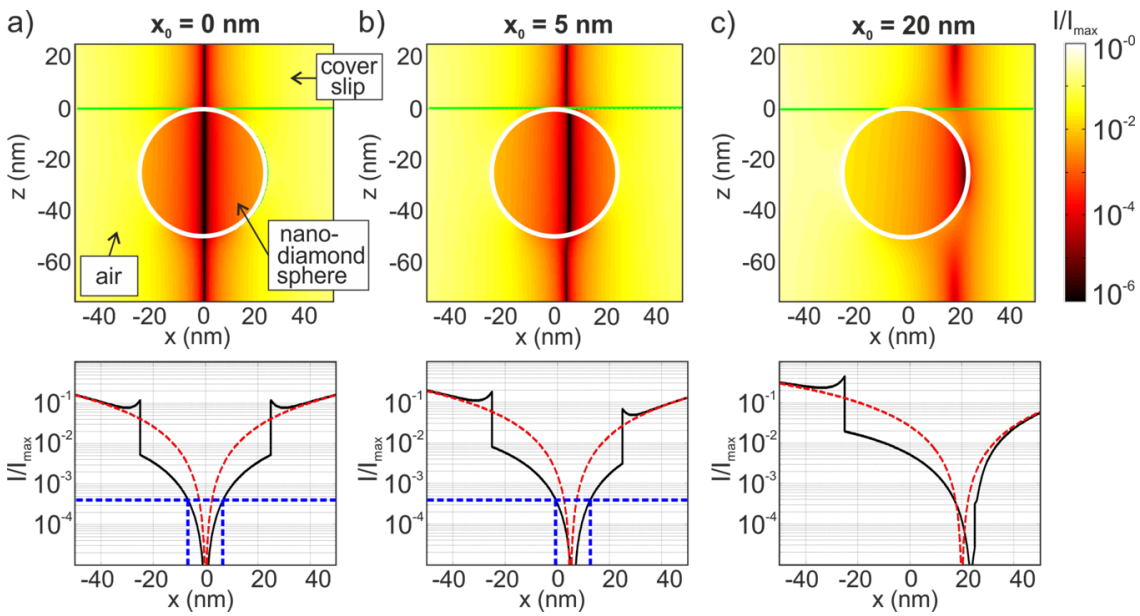

Figure 5. Numerical simulations of the STED intensity distribution of the doughnut beam focused at the interface of a diamond sphere (diameter $50 \mathrm{~nm}$ ) and a glass coverslip marked by the horizontal green line at $z=0 \mathrm{~nm}$. The STED doughnut beam propagates toward the negative $z$ axis. The STED intensity distribution is shown for three different displacements of the STED beam $x_{0}$ with respect to the diamond sphere center: (a) $x_{0}=0 \mathrm{~nm}$, (b) $x_{0}=5 \mathrm{~nm}$, and (c) $x_{0}=20 \mathrm{~nm}$ (intensities are normalized with respect to the intensity at the doughnut rim $I_{\max }$ ). Upper row: STED intensity distribution in the $x-z$ plane cross section through the diamond center. Lower row: STED intensity profiles along the $x$ axis in the sphere center plane $z=-25 \mathrm{~nm}$ (black plain, with diamond sphere; red dashed, reference in air, without sphere). The dashed horizontal blue line corresponds to the critical intensity $I_{c}=4 \times 10^{-4} \cdot I_{\max }$. The distance between the vertical dashed blue line yields a resolution in the $10-15 \mathrm{~nm}$ range.

obtain outstanding resolution performance, we carried out extended numerical simulations. We started by studying the propagation of the STED beam in bulk diamond. The experimentally observed maximum resolution of our STED microscope on NV centers in bulk diamond is around $8 \mathrm{~nm}$. According to our numerical simulations in bulk diamond, the corresponding characteristic STED field intensity at a $4 \mathrm{~nm}$ displacement from the doughnut zero is $I_{\mathrm{c}}=4 \times 10^{-4} \cdot I_{\max }$, where $I_{\max }$ is the STED intensity at the doughnut rim (see Supporting Information). This characteristic intensity can now be employed as a benchmark for the expected STED resolution extracted from analogous simulations of the STED intensity distribution inside nanodiamonds. Figure 5 shows the STED intensity distribution in a spherical nanodiamond with a diameter of $50 \mathrm{~nm}$ for three different displacements of the STED beam with respect to the nanodiamond center. These simulations show that if the doughnut shaped light mode is incident on a diamond nanosphere, the doughnut actually propagates into the particle. Due to the system symmetry, the excitation of the dipolar mode is of vanishing nature as long as the doughnut mode is centered on the ND ${ }^{14,15}$ (see Figure 5a), as opposed to what had formerly been suggested. ${ }^{9}$ Even if the doughnut beam is displaced with respect to the ND center, the dipolar excitation term represents only a minor correction. Instead, the doughnut beam excites mainly higher order electrostatic modes of the ND leading to an effective propagation of the doughnut beam into the ND irrespective of the particle diameter. The simulations presented in Figure 5b,c confirm these theoretical considerations. It is observed that the STED doughnut propagates into the nanodiamond, but the quality of the doughnut zero is slightly compromised, due to the nonvanishing excitation of the dipolar mode. Furthermore, we observe a small shift of the doughnut zero position inside the ND with respect to its position if the ND was absent (cf. black and red curves in lower row plots of Figure $5 a-c$ ).

From Figure $5 \mathrm{a}-\mathrm{c}$ we can extract the expected STED resolution, if we consider the critical intensity $I_{c}$ from the bulk diamond case mentioned above as a benchmark (dashed blue lines in lower row of Figure $5 \mathrm{a}-\mathrm{c}$ ). This way, we find a corresponding STED resolution inside this nanodiamond in the range 10-15 $\mathrm{nm}$. Hence, the application range of STED microscopy and related super-resolution techniques employing doughnut shaped light modes does explicitly include the realm of emitters embedded in subwavelength sized nanoparticles, such as NV centers in nanodiamonds.

As the experimentally investigated nanodiamonds are far from spherical, we performed further simulations for a three-dimensional cylindrical nanodiamond with a circular cross section similar to the ND observed in Figure 3 (ND diameter, $\Delta x=80 \mathrm{~nm}$; height, $\Delta z=75 \mathrm{~nm}$; the STED doughnut propagates in negative $z$ direction). Analogous to the simulations for the sphere, Figure 6 displays the STED intensity profiles on different planes intersecting the diamond cylinder for different STED beam displacements $x_{0}$ with respect to the ND cylinder axis. These simulations clearly show that the STED beam intensity gradient propagates into this nonspherical particle just as well as in the case of the spherical ND; the critical intensity $I_{c}$ yields a STED resolution in the same $10-15 \mathrm{~nm}$ range. Similar to the spherical case, the beam displacement is accompanied 


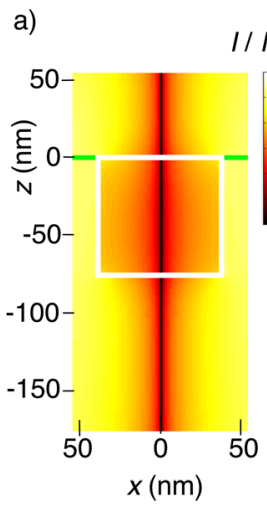

b) $x_{0}=0 \mathrm{~nm}$

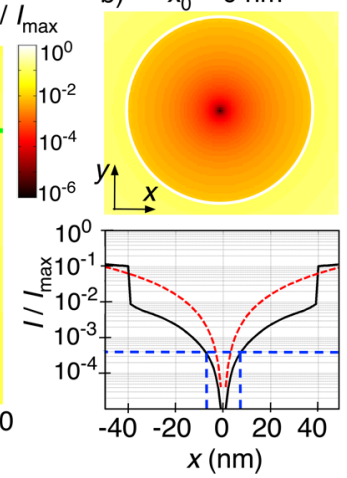

c) $x_{0}=10 \mathrm{~nm}$

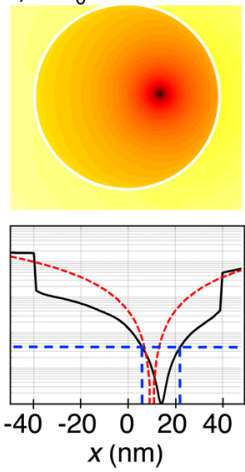

d) $x_{0}=30 \mathrm{~nm}$

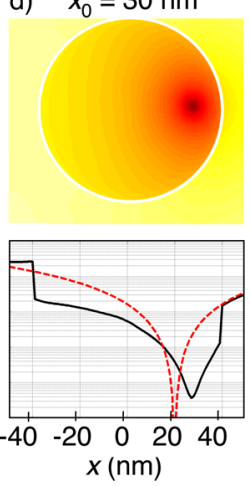

Figure 6. Numerical simulations of the STED intensity distribution of the doughnut beam focused at the interface of a diamond cylinder (height of $75 \mathrm{~nm}$ and diameter of $80 \mathrm{~nm}$ ) and a glass coverslip marked by the horizontal green line at $z=0 \mathrm{~nm}$. The STED beam propagates toward the negative $z$ axis. The STED intensity distribution is shown for three different displacements of the STED beam $x_{0}$ with respect to the cylinder axis: (a and b) $x_{0}=0 \mathrm{~nm}$, (c) $x_{0}=10 \mathrm{~nm}$, and (d) $x_{0}=30 \mathrm{~nm}$ (intensities are normalized with respect to the intensity at the doughnut rim $I_{\max }$ ). (a) STED intensity distribution in the $x-z$ plane cross section through the diamond. (b-d) Upper row: STED intensity distribution in the $x-y$ plane perpendicular to the cylinder axis going through the cylinder center. Lower row: STED intensity profiles along $x$ and in the middle plane at $z=-37.5 \mathrm{~nm}$ (black plain, with diamond cylinder; red dashed, reference in air without cylinder). The dashed horizontal blue line corresponds to the critical intensity $I_{\mathrm{c}}=4 \times 10^{-4} \cdot I_{\mathrm{max}}$. The distance between the vertical dashed blue line yields a resolution in the $10-15 \mathrm{~nm}$ range.

by deformation of the intensity gradient and a slight mismatch of the STED intensity minimum inside the particle with respect to the actual extra-particular doughnut beam position, compromising the fidelity of the NV position in the images to a certain extent. These aberrations are, however, neither specific to the STED technique nor are they a peculiarity in imaging of subwavelength sized particles. Rather, similar aberrations are expected for imaging of micrometer-sized diamond nanospheres independent of the imaging method, i.e., they would equally affect confocal, (semi)stochastic photoswitching and STED-like imaging techniques. ${ }^{16}$

As the maximum and minimum field intensities of both the excitation and STED beam vary a little over the ND volume, the effective resolution is expected to vary slightly across the particle. This effect will also compromise the effective excitation field intensities in confocal and (semi)stochastic photoswitching imaging techniques in direct analogy. Nevertheless, the quality of the STED images and the resulting resolution proved to be very comparable to the case of NV centers in bulk diamond. ${ }^{17}$

\section{CONCLUSION}

We experimentally demonstrated that STED microscopy is capable of imaging single and multiple NV centers inside $40-250 \mathrm{~nm}$ sized nanodiamonds. For all sizes encountered, STED microscopy was able to resolve individual NV centers with a FWHM of
10-20 nm. Our experimental observations are supported by numerical simulations using diamond nanoparticles of diverse sizes and shapes. We did not observe fundamental limitations to the validity of the STED principle for imaging emitters in subwavelength sized nanodiamonds, neither in the simulations nor in the experiment. Hence, individual NV centers can be separated within nanodiamonds with a resolution comparable to NV centers in bulk diamond enabling an individual optical addressing and readout of the spin information which cannot be achieved by means of (semi)stochastic super-resolution techniques. This distinct addressing and readout capability should as well be provided by the generalized STED principle called RESOLFT. ${ }^{18}$ Although the shape and size of the nanodiamonds affect the NV distribution in the image and the effective resolution depends slightly on the NV position inside the nanodiamond, the potential of STED microscopy to discern individual NV centers in nanodiamonds is comparable to that in bulk diamond. ${ }^{17}$ Our conclusion holds for similar objects of investigation, such as fluorophore molecules embedded in a nanoparticle of high refractive index. The far-field optical imaging, targeted addressing and readout of the spin of individual NV centers inside nanodiamonds reported in this study will be highly relevant for their application in quantum sensing and information processing.

\section{MATERIALS AND METHODS}

ND Sample Preparation. The sample was prepared according to previously published protocols: ${ }^{19,20}$ High-pressure hightemperature synthetic diamond nanocrystals from L.M. Van Moppes \& Sons SA (Geneva, Switzerland) with a nitrogen concentration $\approx 100 \mathrm{ppm}$ and a size of $0-250 \mathrm{~nm}$ (type SYP $0-0.25$ ) were irradiated by a $13.6 \mathrm{MeV}$ electron beam for a short time to create vacancies and annealed in vacuum at $800{ }^{\circ} \mathrm{C}$ for $2 \mathrm{~h}$ to favor $\mathrm{N}-\mathrm{V}$ center formation by vacancy migration. This resulted in the creation of $0-20 \mathrm{NV}$ centers per nanodiamond. ${ }^{20}$ The unwanted graphitic layers, which form on the ND surface during 
these first steps, are removed by air oxidation at $550{ }^{\circ} \mathrm{C}$ for $1 \mathrm{~h}$. The resulting oxidized fluorescent NDs were diluted with deionized water to a concentration of about $1 \mathrm{mg} / \mathrm{mL}$. This aqueous suspension was further diluted to a concentration of 1:200 in deionized water and spin coated onto a $210 \mu \mathrm{m}$ thick quartz coverslip. For this study, we used a quartz coverslip featuring a customized, labeled matrix etched into the coverslip surface, which allowed the retrieval of the very same ND on the multiple imaging setups needed. The used ND suspension concentration yielded a mean density of about 0.1 $\mathrm{ND} / \mu \mathrm{m}^{2}$.

STED Microscope Setup for NV Centers. NV centers are excited from the ground state triplet to vibrational sublevels of the first excited triplet state by absorption of a photon from a Gaussian laser beam ( $\lambda_{\text {exc }}=532 \mathrm{~nm}$; pulse length, $55 \mathrm{ps}$; rep. rate, $8 \mathrm{MHz}$ ) having an average power of $60 \mu \mathrm{W}$. Spatially selective inhibition of the occupation of the first excited triplet state was induced by a doughnut-shaped STED laser beam $\left(\lambda_{\text {sted }}=775 \mathrm{~nm}\right.$; pulse length, $2 \mathrm{~ns}$; rep. rate, $8 \mathrm{MHz}$ ) at a maximal time averaged power of around $1 \mathrm{~W}$. Fluorescence was detected in a time-gated fashion. ${ }^{21,22}$

A time intensity autocorrelation detection unit enabled identification of NDs containing just a single NV center by means of their characteristic photon antibunching signature in the fluorescence intensity $I(t)$ time autocorrelation function $g^{(2)}(\tau) \equiv\langle I(0) I(\tau)\rangle /\langle I(0)\rangle^{2,23-25}$ In the pulsed excitation regime, Poissonnian light yields photon correlation peaks that appear at every multiple of the inverse repetition rate of the laser. The absence of the correlation peak at zero delay (Figure $2 c$ ) is the signature of a single emitter.

The dependence of the $\mathrm{NV}^{-}$fluorescence rate on the spin state of the NV center allows extracting information on the $m_{s}$ quantum number. ${ }^{26-28}$ Therefore, the setup featured a loop antenna capable of supplying microwaves, allowing the recording of electron spin resonances (ESR) of the NV ground state triplet on the $m_{\mathrm{s}}=0$ to $m_{\mathrm{s}}= \pm 1$ transition, through detection of the emitted fluorescence. ${ }^{26,29}$ A permanent magnet positioned near the ND sample generated an external magnetic field to lift the degeneracy of the $m_{s}= \pm 1$ states by Zeeman splitting ${ }^{30,31}$ (see Figure $3 e$ ). The magnet position and angle were adapted for each ESR measurement in order to separate the numerous ESR lines optimally. The applied magnetic field varied between 0.02 and $0.04 \mathrm{~T}$.

Numerical Simulations. The simulations were computed based on a finite element method, where the electric field is discretized with edge elements. ${ }^{32}$ The mesh size was adaptative. When modeling the nanosphere, the smallest element had a length of $1.3 \mathrm{~nm}$ and the largest element was $21.3 \mathrm{~nm}$. These values led to solving a linear system with 741000 unknowns.

Conflict of Interest: The authors declare no competing financial interest.

Acknowledgment. We want to thank Prof. Peter van Aken and Felicitas Predel from the STEM facility at the MPI for Intelligent Systems (Stuttgart) for enabling us to record highresolution SEM images, Géraldine Dantelle (Physique de la Matière condensée, École Polytechnique, CNRS, Palaiseau, France) for the preparation of the fluorescent nanodiamond aqueous suspension, and Joseph Lautru for etching the matrix in the coverslips. We thank Gopalakrishnan Balasubramanian, Ronald Walsworth, Loic Rondin, Jean-Philippe Tétienne, Sophie Meuret, Luiz H. G. Tizei and Mathieu Kociak for fruitful discussions. We acknowledged funding from the Volkswagenstiftung, DARPA (QuASAR project), the Agence Nationale de la Recherche (France) under contract number 2010-INTB1002 (NeuroDiam project), and from the Laboratoire d'Excellence NanoSaclay.

Supporting Information Available: Nanodiamond size characterization by AFM and SEM. Correlation between NV number, fluorescence spots and ESR line pairs. STED intensity simulation inside bulk diamond, estimation of STED threshold intensity corresponding to a $8 \mathrm{~nm}$ resolution. This material is available free of charge via the Internet at http://pubs.acs.org.

\section{REFERENCES AND NOTES}

1. Balasubramanian, G.; Chan, I. Y.; Kolesov, R.; Al-Hmoud, M.; Tisler, J.; Shin, C.; Kim, C.; Wojcik, A.; Hemmer, P. R.; Krueger, A.; et al. Nanoscale Imaging Magnetometry with Diamond Spins Under Ambient Conditions. Nature 2008, 455, 648651.

2. Rondin, L.; Tetienne, J.-P.; Spinicelli, P.; Savio, C. D.; Karrai, K.; Dantelle, G.; Thiaville, A.; Rohart, S.; Roch, J.-F.; Jacques, V. Nanoscale Magnetic Field Mapping with a Single Spin Scanning Probe Magnetometer. Appl. Phys. Lett. 2012, 100, 153118.

3. Dolde, F.; Fedder, H.; Doherty, M. W.; Nbauer, T.; Rempp, F.; Balasubramanian, G.; Wolf, T.; Reinhard, F.; Hollenberg, L. C. L.; Jelezko, F.; et al. Electric-Field Sensing Using Single Diamond Spins. Nat. Phys. 2011, 7, 459-463.

4. Kucsko, G.; Maurer, P. C.; Yao, N. Y.; Kubo, M.; Noh, H. J.; Lo, P. K.; Park, H.; Lukin, M. D. Nanometre-Scale Thermometry in a Living Cell. Nature 2013, 500, 54-58.

5. Rittweger, E.; Han, K. Y.; Irvine, S. E. I.; Eggeling, C.; Hell, S. W. STED Microscopy Reveals Crystal Colour Centres with Nanometric Resolution. Nat. Photonics 2009, 3, 144-147.

6. Wildanger, D.; Patton, B. R.; Schill, H.; Marseglia, L.; Hadden, J. P.; Knauer, S.; Schnle, A.; Rarity, J. G.; O'Brien, J. L.; Hell, S. W.; et al. Solid Immersion Facilitates Fluorescence Microscopy with Nanometer Resolution and SubÅngström Emitter Localization. Adv. Mater. 2012, 24, OP309-OP313.

7. Han, K. Y.; Willig, K. I.; Rittweger, E.; Jelezko, F.; Eggeling, C.; Hell, S. W. Three-Dimensional Stimulated Emission Depletion Microscopy of Nitrogen-Vacancy Centers in Diamond Using Continuous-Wave Light. Nano Lett. 2009, 9, 33233329.

8. Tzeng, Y.-K.; Faklaris, O.; Chang, B.-M.; Kuo, Y.; Hsu, J.-H.; Chang, H.-C. Superresolution Imaging of AlbuminConjugated Fluorescent Nanodiamonds in Cells by Stimulated Emission Depletion. Angew. Chem. 2011, 50, 22622265.

9. Greffet, J.-J.; Hugonin, J.-P.; Besbes, M.; Lai, N.; Treussart, F.; Roch, J.-F. Diamond Particles as Nanoantennas for Nitrogen-Vacancy Color Centers. 2011, No. arXiv:1107.0502. arXiv.org e-Print archive.

10. Gu, M.; Cao, Y.; Castelletto, S.; Kouskousis, B.; Li, X. SuperResolving Single Nitrogen Vacancy Centers within Single Nanodiamonds Using a Localization Microscope. Opt. Express 2013, 21, 17639-17646.

11. Fölling, J.; Bossi, M.; Bock, H.; Medda, R.; Wurm, C. A.; Hein, B.; Jakobs, S.; Eggeling, C.; Hell, S. W. Fluorescence Nanoscopy by Ground-State Depletion and Single-Molecule Return. Nat. Methods 2008, 5, 943-945.

12. Chen, E. H.; Gaathon, O.; Trusheim, M. E.; Englund, D. WideField Multispectral Super-Resolution Imaging Using SpinDependent Fluorescence in Nanodiamonds. Nano Lett. 2013, 13, 2073-2077.

13. Davies, G.; Hamer, M. F. Optical Studies of the $1.945 \mathrm{eV}$ Vibronic Band in Diamond. Proc. R. Soc. London, Ser. A 1976, 348, 285-298.

14. Mojarad, N. M.; Agio, M. Tailoring the Excitation of Localized Surface Plasmon-Polariton Resonances by Focusing Radially-Polarized Beams. Opt. Express 2009, 17, 117-122.

15. Zhang, W.; Gallinet, B.; Martin, O. J. F. Symmetry and Selection Rules for Localized Surface Plasmon Resonances in Nanostructures. Phys. Rev. B 2010, 81, 233407.

16. Hell, S.; Reiner, G.; Cremer, C.; Stelzer, E. H. K. Aberrations in Confocal Fluorescence Microscopy Induced by Mismatches in Refractive Index. J. Microsc. 1993, 169, 391405.

17. Pezzagna, S.; Rogalla, D.; Wildanger, D.; Meijer, J.; Zaitsev, A. Creation and Nature of Optical Centres in Diamond for Single-Photon Emission-Overview and Critical Remarks. New J. Phys. 2011, 13, 035024.

18. Hell, S. W. Far-Field Optical Nanoscopy. Science 2007, 316, 1153-1158.

19. Dantelle, G.; Slablab, A.; Rondin, L.; Laine, F.; Carrel, F.; Bergonzo, P.; Perruchas, S.; Gacoin, T.; Treussart, F.; Roch, J.-F. Efficient Production of NV Colour Centres in 
Nanodiamonds using High-Energy Electron Irradiation. J. Lumin. 2010, 130, 1655-1658.

20. Rondin, L.; Dantelle, G.; Slablab, A.; Grosshans, F.; Treussart, F.; Bergonzo, P.; Perruchas, S.; Gacoin, T.; Chaigneau, M.; Chang, H.-C.; et al. Surface-Induced Charge State Conversion of Nitrogen-Vacancy Defects in Nanodiamonds. Phys. Rev. B 2010, 82, No. 115449.

21. Moffitt, J. R.; Osseforth, C.; Michaelis, J. Time-Gating Improves the Spatial Resolution of STED Microscopy. Opt. Express 2011, 19, 4242-4254.

22. Vicidomini, G.; Schnle, A.; Ta, H.; Han, K. Y.; Moneron, G.; Eggeling, C.; Hell, S. W. STED Nanoscopy with Time-Gated Detection: Theoretical and Experimental Aspects. PLoS One 2013, 8, e54421.

23. Hanbury Brown, R.; Twiss, R. Q. The Question of Correlation between Photons in Coherent Light Rays. Nature 1956, $178,1447-1448$.

24. Carmichael, H. J.; Walls, D. F. Proposal for the Measurement of the Resonant Stark Effect by Photon Correlation Techniques. J. Phys. B: At. Mol. Phys. 1976, 9, L43.

25. Kimble, H. J.; Dagenais, M.; Mandel, L. Photon Antibunching in Resonance Fluorescence. Phys. Rev. Lett. 1977, 39, 691-695.

26. Gruber, A.; Drbenstedt, A.; Tietz, C.; Fleury, L.; Wrachtrup, J.; Borczyskowski, C. Scanning Confocal Optical Microscopy and Magnetic Resonance on Single Defect Centers. Science 1997, 276, 2012-2014.

27. Harrison, J.; Sellars, M.; Manson, N. Optical Spin Polarisation of the N-V Nentre in Diamond. J. Lumin. 2004, 107, 245-248. Proceedings of the 8th International Meeting on Hole Burning, Single Molecule, and Related Spectroscopies: Science and Applications.

28. Manson, N. B.; Harrison, J. P.; Sellars, M. J. Nitrogen-Vacancy Center in Diamond: Model of the Electronic Structure and Associated Dynamics. Phys. Rev. B 2006, 74, 104303.

29. Jelezko, F.; Gaebel, T.; Popa, I.; Gruber, A.; Wrachtrup, J. Observation of Coherent Oscillations in a Single Electron Spin. Phys. Rev. Lett. 2004, 92, 076401.

30. Maze, J. R.; Stanwix, P. L.; Hodges, J. S.; Hong, S.; Taylor, J. M.; Cappellaro, P.; Jiang, L.; Dutt, M. V. G.; Togan, E.; Zibrov, A. S.; et al. Nanoscale Magnetic Sensing with an Individual Electronic Spin in Diamond. Nature 2008, 455, 644-647.

31. Taylor, J. M.; Cappellaro, P.; Childress, L.; Jiang, L.; Budker, D.; Hemmer, P. R.; Yacoby, A.; Walsworth, R.; Lukin, M. D. High-Sensitivity Diamond Magnetometer with Nanoscale Resolution. Nat. Phys. 2008, 4, 810-816.

32. Bossavit, A. A Rationale for 'Edge-Elements' in 3-D Fields Computations. IEEE Trans. Magn. 1988, 24, 74-79. 